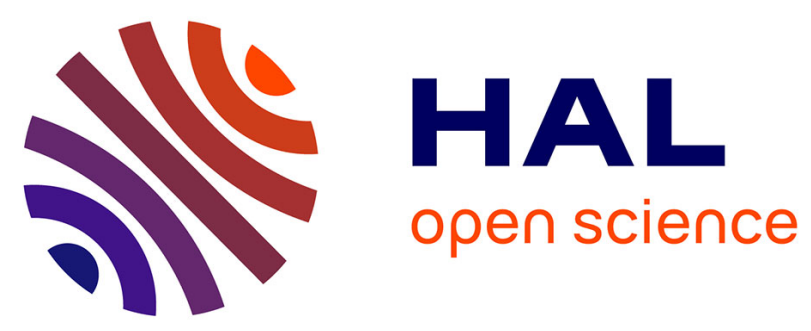

\title{
A breakthrough for prepaid payment: End to end token exchange and management using secure SSL channels created by EAP-TLS smart cards
}

Pascal Urien, Marc Pasquet, Christophe Kiennert

\section{- To cite this version:}

Pascal Urien, Marc Pasquet, Christophe Kiennert. A breakthrough for prepaid payment: End to end token exchange and management using secure SSL channels created by EAP-TLS smart cards. CTS, 2011, United States. pp.476 - 483, 10.1109/CTS.2011.5928726 . hal-00673667

\section{HAL Id: hal-00673667 \\ https://hal.science/hal-00673667}

Submitted on 24 Feb 2012

HAL is a multi-disciplinary open access archive for the deposit and dissemination of scientific research documents, whether they are published or not. The documents may come from teaching and research institutions in France or abroad, or from public or private research centers.
L'archive ouverte pluridisciplinaire HAL, est destinée au dépôt et à la diffusion de documents scientifiques de niveau recherche, publiés ou non, émanant des établissements d'enseignement et de recherche français ou étrangers, des laboratoires publics ou privés. 


\section{A Breakthrough for Prepaid Payment: End to End Token Exchange and Management Using Secure SSL Channels Created by EAP-TLS Smart Cards}

\author{
Pascal Urien \\ Telecom ParisTech \\ 23 av. d'italie, Paris, France \\ pascal.urien@telecom- \\ paristech.fr
}

\author{
Marc Pasquet \\ GREYC CNRS UMR 6072 \\ laboratory, Higher Education \\ National Engineering School \\ of Caen, France \\ marc.pasquet@ensicaen.fr
}

\author{
Christophe Kiennert \\ EtherTrust \\ 62bis rue Gay Lussac, Paris, \\ France \\ christophe.kiennert@ethertru \\ st.com
}

\begin{abstract}
In this paper we present an innovative architecture for prepaid services. Digital tokens are securely exchanged between EAP-TLS smart cards used both by merchant and customer. We describe the global framework that comprises a back-office server delivering tokens, a frontoffice server collecting tokens from merchant terminal, merchants and customers equipped with smart cards. We detail data exchange choreography and discuss performances issues for the experimental platform built with commercial devices.
\end{abstract}

KEYWORDS: Security, EMV, TLS, WEB, Smart Card

\section{INTRODUCTION}

Prepaid facilities refer to services bought in advance; from a legal and practical point of view, they are very different from fiduciary money.

Fiduciary money (coming from the Latin fiducia meaning confidence or trust), is typically a paper certificate including some typographic authenticity proofs (banknote...) used to acquire any goods. As an illustration the US dollar could be converted in gold until 1971.

Prepaid services (see [1] for a more detailed analysis) target a specific class of consumer goods, which have been (pre) paid before their purchase.

We divide prepaid systems in two classes, centralized and distributed.
Centralized architectures, such as phone prepaid calls [14], deal with serial numbers, typically printed on vouchers, and hidden by a scratch area. The serial number lifecycle is managed by a database, whose interface may be a RADIUS server. The user is registered in the system; his account is credited with the value associated with the serial number, and afterwards is managed by on-line facilities.

In distributed infrastructures, prepaid transactions are performed off-line. The customer generally gets a voucher, with a serial number and some typographic authenticity proofs. The serial number may include cryptographic digits, typically computed with a signature algorithm. As an illustration Cheque Dejeuner, a French company specialized in prepaid services, delivered (in 2006) vouchers to 15 millions of customers, for an amount of 2,6 billions $€$.

In this paper we present a distributed architecture that aims at replacing printed tickets by digital tokens, securely exchanged [5] between smart cards. These tamper resistant devices are widely used for electronic payments, mainly in the EMV framework [4]; they are also deployed as electronic purse [15] in Europe, for micro-payments (a few $€$ ) operations.

The central idea of our proposal is to establish secure SSL tunnels between smart cards, or between smart cards and SSL servers. SSL [8] [9] is the de facto standard for the Internet security; it is the cornerstone of the e-Commerce. Digital tokens are provisioned from back office servers. They are exchanged via customers and merchant smart cards. They are cleared through sessions between merchant smart cards and front office servers. 
This paper is organized according to the following plan. Section two introduces payment systems. Section three unveils our new prepaid framework. Section four presents EAP-TLS smart cards and performances issues. Finally section five concludes this paper.

\section{ABOUT PAYMENT SYSTEMS}

According to [1] "Commerce always involves a payer and a payee - who exchange money for goods of services and at least one financial institution - who links bits to money". Generally two financial entities are involved in a transaction, an issuer acting for the payer, and an acquirer used by the payee (frequently refereed as the merchant).

\subsection{About card transaction}

A payment card transaction usually consists of two steps [2], transaction flow and clearing and settlement. Depending on the card type payer and payee may be credited / debited according to different policies.

The transaction flow is used for authorization purpose. Merchant-acquirer and issuer bank are connected via a secure payment card network (PCN) managed by card companies. Some of them (like American Express) may also play the role of acquirers. The merchant collects information about the card (number, date of expiry, type, etc.) via a terminal device, and sends it to the acquirer. This latter forwards these data (but this is not always mandatory) to the issuer financial institution (through the $\mathrm{PCN}$ ) that checks the account status. A response is returned to the acquirer, which upon success, delivers an authorization code to the merchant terminal.

The Clearing and Settlement process deals with payment operations and funds transfer, typically occurring within a few days.

\subsection{Security Issues}

The payment card industry data security standard (or $P C I$ $D S S[3])$, specifies security requirements dispatched in six main groups.

1- Build and Maintain a Secure Network, by deploying secure physical infrastructure (firewall...) and logical protections (Virtual Private Networks...).

2- Protect Cardholder Data, by using cryptographic means (strong encryption...) for data storage and transmission.
3- Maintain a Vulnerability Management Program, by deploying anti-virus software, updating operating systems with security patches, developing secure WEB applications.

4- Implement Strong Access Monitor Control Measures, by avoiding passwords for access control and using two factors authentications tokens or biometric means, especially for operations dealing with cardholder data.

5- Regularly Monitor and Test Networks, by tracking and monitoring accesses to the network and by regularly testing system security and processes.

6- Maintain Information Security Policy, which targets information security for both employees and contractors.

\subsection{The EMV Use Case.}

EMV acronym comes from Europay MasterCard and Visa, the three companies that in 1994 initiated this standard [4]. Today most of electronic payments are performed with EMV cards. In 2009 more than 944 million EMV compliant chip-based payment cards were deployed in the world.

In non electronic payment, card information (number, validity date...) is read from the magnetic strip. In the EMV technology a tamper resistant micro-controller (a smart card), stores cardholder data, but also supports additional security features.

The card is equipped with an issuer certificate, and contains a signature (called the SSAD, Signed Static Application Data) of the embedded information, computed with the associated private key.

The device optionally includes an individual certificate (delivered by the issuer), and a private key, which proves the electronic chip authenticity thanks to a challenge/response mechanism, called DDA (dynamic authentication).

The EMV card generates application cryptograms (AC) generally based on a triple DES algorithm working with a 112 bits secret key.

A transaction is initiated by the merchant terminal, via a GENERATE AC command (ARQC, Authorization Request Cryptogram), conveying various parameters such as the amount or the date. Upon success, the device returns a cryptogram data information (CDI).

The merchant forwards an authorization request to the acquirer that comprises the ARQC, the CDI, and 
additional attributes. Depending on the response, the terminal indicates its decision to accept or decline the transaction in a second GENERATE AC command.

The card generates a Transaction Certificate (TC) for an approval or an Application Authentication Cryptogram (AAC) for a decline.

The merchant transmits this notification to the acquirer via a confirmation message.

The datagram collected by the first GENERATE AC command, indicates if a PIN was successfully given by the cardholder. This mechanism enforces a dual factor authentication, i.e. a cryptographic key stored in the chip and a PIN known by a human user.

\subsection{Prepaid Systems}

As mentioned by [1] in prepaid systems, "a certain amount of money is taken away from the payer, before purchases are made". This mode of operation works for electronic purse and bank checks.

\section{A NEW PREPAID FRAMEWORK}

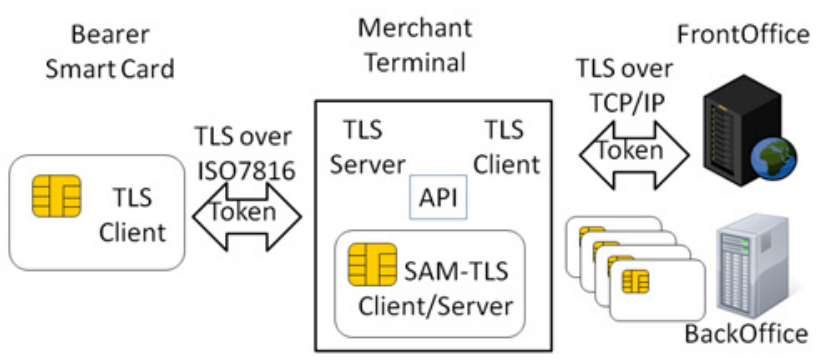

Figure 1. Main Components

\subsection{Overview}

The customer generally gets about twenty vouchers for a month. Each has a serial number, a value date, a value (e.g. $7.5 €$ ) and some typographic authenticity proofs. The serial number may include cryptographic digits, typically computed with a signature algorithm. The Front End issues the vouchers by delegation of the Back End. These vouchers are sent to the card, by the way of a secure channel and are stored in the chip, through the terminal. Then the card is able to use these vouchers, but only one or two each time. The voucher(s) is (are) sent by the card to the Merchant terminal, by a secure channel, to be stored in the chip of the SAM (Secure Access Module). At the end of the day, the different vouchers stored during the day in the SAM are collected by the Front End by a secure channel, verified and destroyed. The value is used to credit the merchant through the Back End.

\subsection{Bearer}

The cardholder belongs to a company that offers him a value added service we can call "lunch voucher". This voucher is paid half by the company, half by the bearer. It can be used to pay a part, or the totality, of the bearer's lunch. The bearer's company buys the vouchers to a specialized company as Cheque Dejeuner, in Europe. The former system delivered these prepaid vouchers in paper checks. They were sent to the company and given to the bearer who may use no more than two vouchers per day. The vouchers are accepted as lunch payment in a large number of European restaurants.

We have designed the new electronic architecture to be as close as possible to the former one, for two main reasons: in one hand to protect the value added chain of the specialized company, and in another hand not to confuse the bearers.

The bearer could use an Internet connection to know how many tokens are still available.

\subsection{Smart Card}

The smart card is issued by the specialized company from its Back End and send to the Bearer's company which delivers it to the cardholder. This Smart Card is empty and has to be inserted in the Merchant terminal to be filled with tokens. A technical description of the card is given in $\S 4.1$.

\subsection{Tokens}

Tokens are issued by the Front End, which gets a list of tokens to issue from the Back End. The life cycle of the token is: existence in the Back End, creation in the Front End, life in the bearer's Smart card or in the Merchant's SAM, destruction in the Front End and trace of existence in the Back End.

The token is made of: an X509 certificate and a record containing the voucher value (the bearer's company can choose any values for the voucher from $1 €$ up to $20 €$ ) in clear and seal in the certificate. Thus, the value can be read by the merchant terminal and compared in the Front End after the daily collection.

Tokens are stored only in secure devices: Smart card, SAM, HSM (Hardware Security Module). 


\subsection{Merchant Terminal}

The merchant terminal dialogs with the bearer's card to accept the payment with tokens. The terminal verifies the value date, the value of the token and gets one or two tokens as ordered by the terminal dialog with the bearer. The PIN code is not necessary at this level. This terminal is contactless for payments by tokens and uses its contact capability to fill the card with tokens (when the card is empty, the cardholder enters his PIN code, the terminal calls the Front End and lets the smart card create a secure channel to transfer the tokens from the Front End to the smartcard).

The merchant terminal assures the transmission flow: from the smartcard to the SAM plugged under the terminal, from this SAM to the Front End, from the Front End to the smartcard as shown in figure 2 .

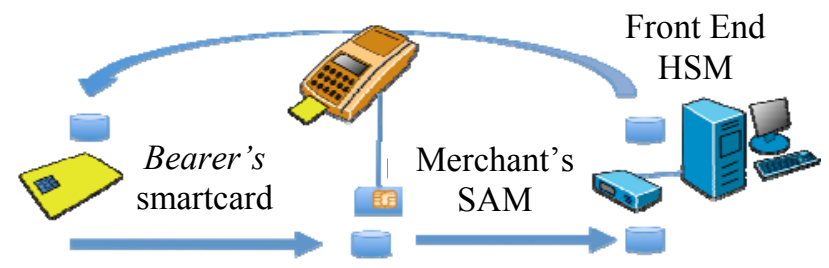

Figure 2. Token exchanges

A phone line, a GSM line or an Internet connection, connects the Merchant terminal to the Front End either.

Today the terminals are specific but in the future software will be included in EMV POS to limit the implementation costs.

\subsection{Issuer/Acquirer Front End}

The Front End is:

- Issuer of tokens. In fact, the Front End is connected to a Hardware Security Module. This HSM creates tokens, which are stored encrypted in the Database of the Front End (FE). Only the registered cards can be fulfilled by the corresponding Tokens (cards and tokens belong to the bearer's company).

- Acquirer of Merchant's Terminal data. The terminal phone calls one time each day the FE to perform the collection of tokens stored in the SAM. The FE can collect only registered terminals.

If the verification of the token is valid, the FE destroys the different tokens and advises the Back End to credit the merchant account.

Security software manages fraud prevention.

\subsection{Issuer Back End}

The Back End (BE):

- Issues the different cards: bearer's cards and SAM, as well as the PIN code (the cards are personalized with the files issued from the Back End)

- Manages the different flows: token creation, accounts credit and debit.

A specific Web access for the Bearer, the Bearer's Company and the Merchant is interfaced from the Back Office (the Back End is considered as a part of the Back Office of the specific company).

\section{EAP-TLS smartcards}

An EAP-TLS [11] [12] [13] device establishes a secure TLS tunnel between the bearer and the merchant terminal. The card located in the terminal is called the Secure Access Module (SAM). It supports server and client resources, is used first to collect tokens from the customer, and second to backup these elements to the front office server.

\subsection{About Smartcards and EAP-TLS Devices.}

A smart card [5] is a tamper resistant microcontroller, whose size is about $25 \mathrm{~mm}^{2}$, which includes a $\operatorname{CPU}(8,16$, 32 bits), and various type of memories such as ROM (a few hundred KB), RAM (about $10 \mathrm{~KB}$ ), and non volatile memory (FLASH or E2PROM, about $128 \mathrm{~KB}$ ). Security is enforced by multiple hardware and software countermeasures. About 5 billions of these electronics chips were produced in 2010, for telecommunication applications (SIM cards, $70 \%$ of the market) or financial transactions (EMV cards, $20 \%$ of the market). Most of these components run a Java Virtual Machine (JVM) [6], and therefore may execute applications written for Javacards with a subset of the Java language.

The SSL protocol (and TLS, a modified version standardized in 1999 by the IETF) is the cornerstone for secure exchanges over the Internet [8] [9]. Everyday billions of net surfers establish protected HTTP sessions for various purposes such as eMail reading, or electronic commerce transactions. This protocol has been deeply analyzed by the research community and enforces strong security; it supports a wide range of cryptographic algorithms such as asymmetric calculations for key exchanges (RSA, DH, ECC...), symmetric functions for data privacy ( $\mathrm{RC} 4,3 \mathrm{xDES}, \mathrm{AES})$ and hash procedures (MD5, SHA1, SHA256) for ephemeral keys generation. 
Although TLS messages are usually shuttled by the TCP transport, a new protocol (EAP-TLS) was invented in 1999 [10] in order to establish TLS sessions without TCP flavors, according to a datagram paradigm.

EAP-TLS smartcards [11] [12] [13] were initially designed for access control in Wi-Fi networks, in an IEEE $802.1 \mathrm{x}$ infrastructure. They may be used both on the client terminal, and on the authentication server side (typically a RADIUS server); they dialog through EAP packets and setup TLS tunnels.

EAP-TLS applications are written for javacards, the code sizes are respectively $20 \mathrm{~KB}$ and $25 \mathrm{~KB}$ for client only and client/server applet.

\subsection{Performances Issues}

A TLS session is opened according to two different modes: full and resume.

A full session is opened via a four way handshake; it usually deals with PKI facilities. The server delivers his certificate (and those of the Certification Authority), the client computes a PreMasterKey and forwards it encrypted with the server public key (found in its certificate). Optionally, but in our case we always implement this facility, the client is equipped with a X509 certificate sent to the server and authenticated by a signature generated with the client private key.

A resume (or abbreviated session) re-uses a previously computed MasterSecret by a full session. It works according to a three ways handshake and only deals with symmetric cryptographic procedures.

We need to perform a transaction between the cardholder and the merchant terminal in an acceptable amount of time, five seconds seems a realistic limit.

EAP-TLS applications are written for javacards; these components are equipped with crypto-processors offering RSA facilities, but hash functions are provided by procedures executed by a modest CPU.

\begin{tabular}{|c|c|c|c|c|c|c|}
\hline RSA & RSA & MD5 & SHA1 & $3 \times \mathrm{xDES}$ & AES & RC4 \\
\hline Pub & Priv & /bloc & /bloc & /bloc & /bloc & /byte \\
\hline 1024 & 1024 & $64 \mathrm{~B}$ & $64 \mathrm{~B}$ & $8 \mathrm{~B}$ & $16 \mathrm{~B}$ & \\
\hline 25 & 550 & 0,50 & 0,90 & 2,10 & 2,60 & 0,50 \\
\hline
\end{tabular}

Figure 3. Computing Performances (in ms)

Basic cryptographic performances are illustrated by figure 3 , for the best Javacard available on the market that we could test. Furthermore we notice a data throughput of about 6000 Bytes/s.

The opening of a full session based on 1024 bits RSA cryptography, requests $2,0 \mathrm{~s}$. It exchanges 2,500 bytes, whose transfer costs 0,40s. About 230 MD5 and SHA1 calculations are performed (dealing with 64 bytes blocs), which consumes 0,32s. One RSA operation with the private key, and two RSA procedures with public keys, are computed in $0,60 \mathrm{~s}$. The sum of the previously cited operations is $1,32 \mathrm{~s}$; the remaining time $(0,68 \mathrm{~s}=2,00-$ $1,32)$ is burnt by the java code execution.

The opening of a resume session costs $0,50 \mathrm{~s}$. It requires the exchange of 250 bytes $(0,04 \mathrm{~s})$, and the calculation of $75 \mathrm{MD} 5$ and SHA1 that consumes $0,11 \mathrm{~s}$. The remaining time $(0,35 \mathrm{~s})$ is needed by the java code execution.

Because both the customer and the merchant terminal use an EAP-TLS device, a session opening costs $4,0 \mathrm{~s}$ or $1,0 \mathrm{~s}$ depending on the operating mode either full or resume.

Once the secure TLS channel has been established, messages exchanged by the client and the server are encrypted with the selected algorithm and integrity is enforced by a HMAC procedure. According to figure 3, we observe that the computing time is mostly consumed by encryption and decryption operations (in other word hash calculations have a second order effect). As an illustration, for a classical RC4, HMAC-MD5 cipher suite, the transfer of 1000 bytes burnt $500 \mathrm{~ms}$ for encryption, $16 \mathrm{~ms}$ for MD5 hash, and 170ms for data transmission.

\subsection{A Transaction Scenario}

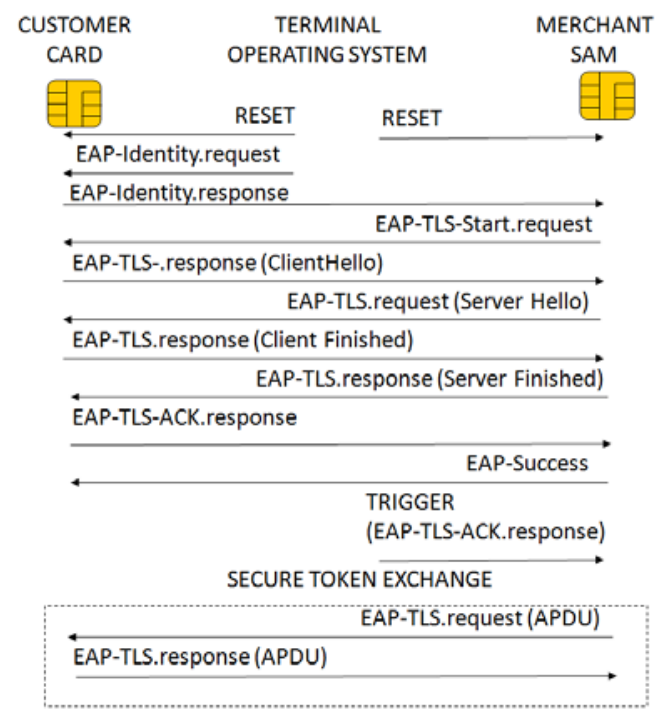

Figure 4. A Transaction Scenario 
As described in [11], EAP messages are transported by ISO7816 [5] packets whose maximum size is about 256 bytes; consequently a segmentation/reassembly process is managed by the ISO7816 driver.

When the merchant terminal starts a new transaction (see annex I for packets details) it performs a reset both on client and SAM cards (see figure 4).

Afterwards according to the EAP standard it sends an EAP-Identity.request message to the client, which returns an EAP-Identity.response, forwarded to the server.

This later produces an EAP-TLS-Start.request packet that notifies the beginning of an EAP-TLS session.

Thereafter TLS messages are shuttled in EAP-TLS packets, the client receives requests and transmits responses, while the server works in a symmetric way.

Upon success, the server forwards a last packet, called EAP-Success, which indicates that a new SSL session has been opened.

At this step client and server devices are ready to exchange encrypted information in EAP-TLS packets.

The terminal delivers a ServerTrigger message, expressed as an EAP-TLS-ACK.response. The SAM builds a command encrypted according to the negotiated CipherSuite, and encapsulated in an EAP-TLS.request packet.

Thereafter commands used for token exchange are encapsulated by EAP-TLS requests (delivered by the server) and responses (produced by the client), transparently conveying TLS messages.

\subsection{Connection to the Front End server}

This scenario (see figure 5) occurs when the SAM needs to backup previously collected tokens to the front office. Packets are detailed in annex II.

The terminal manages the TCI/IP connectivity thanks to the well know sockets API. It opens a TCP session with the remote Front Office server on the port 443.

It sends a Reset command to the SAM, and an EAP-TLSStart.request packet indicating the beginning of the EAPTLS session. The SAM returns an EAP-TLS.response packet transporting the first TLS message (the TLS Client Hello Message).
A software bridge converts EAP-TLS.response packets in outgoing TLS messages (forwarded to the remote server), and ingoing TLS messages (received from the remote server) in EAP-TLS.request packets (sent to the SAM)

If a TLS session is successfully opened, the terminal sends a ClientTrigger message, expressed as an EAP$T L S$-Ack.request packet. The SAM builds a command, encrypted according to the negotiated CipherSuite, and encapsulated in an EAP-TLS.response.

Afterwards the software bridge transforms EAP-TLS response in a TLS message, and a secure dialog occurs between the SAM and the Front Office in order to extract tokens.

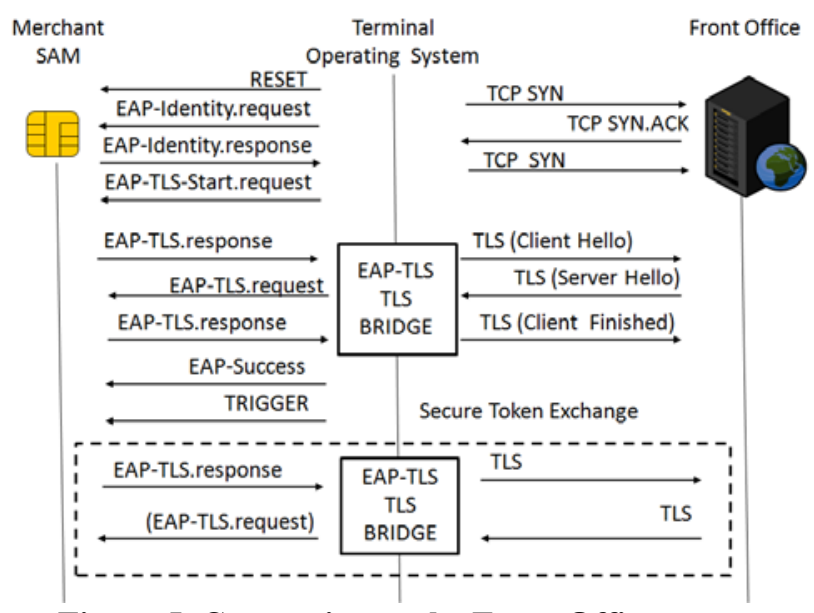

Figure 5. Connection to the Front Office server

\section{CONCLUSION}

In this paper we have presented an innovative architecture for prepaid services. Experimental platform demonstrated that performances are compatible with real time constraints. The next step targets the deployment of this system for a set of beta customers.

We strongly thank Cheque Dejeuner Group for their help. The deployment will be realized on a set of their customers.

\section{ANNEX I}

This annex illustrates an EAP-TLS dialog, in the resume mode between the client and the SAM. EAP-TLS header is underlined, TLS message is in bold.

Commands sent to smart cards are called APDUs, and are defined by the ISO7816 standard. Request messages have a prefix, whose length is five bytes, respectively noted 
CLA INS P1 P2 P3. The P3 attribute is the size of the body, or the size of the requested information. Response messages have an optional body, and end by a two bytes status; the hexadecimal value 9000 indicates a successful operation. EAP-TLS messages [10] begin by a four bytes prefix noted, code, identifier, and length. Code defines the class of the message: 01 for request, 02 for response and 03 for success Identifier is a label used both for requests and responses. The length attribute is the EAP message size. The fifth byte indicates the type of operation, 01 is used for Identity and $0 \mathrm{~B}$ for EAP-TLS. The sixth byte (the flag attribute) gives more details about the EAP-TLS packet structure. The hexadecimal value 80 is followed by the content size coded by a 32 bits number; a null value means that no options are used. The content of an EAP-TLS packet is a TLS message whose format is precisely detailed in [9].

The Terminal sends Reset to the Client.

Tx: A0 19100000

Rx: 9000

The Terminal sends Reset to the SAM.

Tx: A0 19100100

Rx: 9000

The Terminal sends EAP-Identity.request to the Client. Tx: A0 $80000005 \underline{01 \text { A5 } 000501}$

The Terminal sends EAP-Identity.response to the SAM. Tx: A0 800000 0B 02 A5 00 0B 0163 6C 6965 6E 74

The SAM sends EAP-TLS-Start.request to the Terminal. Rx: 01 A6 0006 0D 209000

The Terminal sends EAP-TLS.response to the SAM. Tx: A0 800000 5C 02 A6 00 5C 0D 800000005216 030100 4D 010000490301 4D 08 FA F0 06 D4 E5 C2 8074 D6 5454 3D 9604 6D D1 A9 4D 8B 98 AF 5C 49 8D EB 1A C8 96 5A 42205725 5C 8171 1779 C8 E1 44 1A 51 6E FD 532132 EC 2D 07 F8 75963690 DA BC 3745 B1 9B C0 0002000401 00

The SAM sends EAP-TLS.request to the Terminal, Rx: 01 A7 0084 0D 80000000 7A 16030100 4A 02 0000460301 4D 08 FA F0 6C AC 27 C3 26 C8 0E 46 F7 8408 A6 45 B8 EC 0D 573793 4C 53 E5 01 19831725 E3 20 57 25 5C 81711779 C8 E1 44 1A 51 6E FD 532132 EC 2D 07 F8 75963690 DA BC 3745 B1 9B C0 00040014030100010116 03010020 A7 3D 21 C8 9895 A1 B6 ED 74 C2 F0 D1 9D 04 E0 75 DC C1 CF 67 OA 0E B3 9B 9087 CD A6 64 BF AD 9000
The Terminal sends EAP-TLS.response to the SAM. Tx: A0 8000003502 A7 0035 0D 80000000 2B 14 03010001011603010020 B9 3C 0309 E1 F9 A 60 C0 D9 F3 2851 DF D5 6F 66296023 5A 10 0A E1 A1 67 A2 F9 707381 C7

The SAM sends EAP-Success to the Terminal. Rx: 03 A7 00049000

The Terminal sends the Server Trigger to the SAM. Tx: A0 8000000602 A8 $00060 \mathrm{D} 00$

The SAM sends EAP-TLS.request to the Terminal. One data byte is sent encrypted and HMACed by the SAM. Rx: 01 A9 04 1F 0D 80000000161703010011 AB 6C B3 96 B4 D9 59 2B 0394 9F C6 13 F2 E4 D1 81 9000

\section{ANNEX II}

This annex illustrates a TLS connection, in the resume mode, between the SAM and the Front Office server. EAP-TLS header is underlined, TLS message is in bold.

The Terminal sends Reset to the SAM.

Tx: A0 19100000

Rx: 9000

The Terminal sends EAP-Identity.request to the SAM. Tx: A0 8000000501 A5 000501

The SAM sends EAP-Identity.response to the Terminal. Rx: 02 A5 000 0B $01636 \mathrm{C} 69656 \mathrm{E} 74$

The Terminal sends EAP-TLS-Start.request to the SAM. Tx: A0 800000 0A 01 A6 0006 0D 20 6E 0716 4D

The SAM sends EAP-TLS.response to the Terminal. Rx: 02 A6 00 5C 0D 800000005216030100 4D 01 0000490301 6E 0716 4D 0F FE F3 7729 E7 B2 7502 F7 BE 89 6C CB CB 52 15 9A CB EC 9B 38 C7 F9 3E 45 E8 3220 FC F1 46 8E 74 CF F7 441778 9B A2 C5 C9 A1 97 A9 2D BC D1 58 7B 0B BC 6373 AA 2A EA CE 97880002000401009000

The Terminal sends EAP-TLS.request to the SAM.

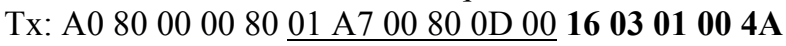
020000460301 4D 1607 6E E7 AB C0 25 F6 E5 0B DA 9749 7D 31 0F 6E 9F 3F EA FD 6875 CB E8 5086 A5 27 8B 0E 20 FC F1 46 8E 74 CF F7 4417 78 9B A2 C5 C9 A1 97 A9 2D BC D1 58 7B 0B BC 63 73 AA 2A EA CE 9788000400140301000101 1603010020 C6 A3 AF 49 D2 8E 0697 2A 94 6C 4C 9D 69 A5 DA 9D 2941 DB 573147237242 C4 0F 0A 8A FD 66 
The SAM sends EAP-TLS.response to the Terminal.

Rx: 02 A7 0035 0D 80000000 2B 140301000101 160301002040 F9 AF FF D9 E9 4F $8 D 5824$ D1 A9 6736 D1 34 C2 F3 D7 EC 56 D3 46 F7 9E D7 5B 3243 7F D9 E0 9000

The Terminal sends the trigger command (EAP-TLS$A C K$.request) to the SAM.

Tx: A0 $80000006 \underline{01 \text { A8 } 0006 \text { 0D 00 }}$

The SAM sends EAP-TLS-ACK.response to the Terminal, indicating that no data are available.

Rx: 02 A8 00 0A 0D 80000000009000

The Terminal sends EAP-TLS.request to the SAM, including four bytes of data, HMACed by the TLS server. Tx: A0 8000002101 A9 00 21 0D 00 1703010016

E2 F1 9092 A8 87 D3 0E CC 9E 41 DD 3977 0E 7A 39 D6 E8 ED 30 4A

The SAM sends EAP-TLS.response to the TLS server, including four bytes of data, HMACed by the SAM. Rx: 02 A9 0025 0D 80000000 1B 1703010016 CA 2C B8 9F 8A 43 A7 33 D0 2E DA 76 OD 27 A8 0A 14 A6 02 25 3E 199000

\section{REFERENCES}

[1] Asokan, N.; Janson, P.A.; Steiner, M.; Waidner, M.; "The state of the art in electronic payment systems", Computer Volume: 30, Issue: 9, 1997

[2] Jing Liu; Yang Xiao; Hui Chen; Ozdemir, S.; Dodle, S.; Singh, V.; "A Survey of Payment Card Industry Data Security Standard", Communications Surveys \& Tutorials, IEEE Volume: 12, Issue: 3, 2010

[3] PCI Security Standard Council, "Payment Card Industry Data Security Standard", http://www.pcisecuritystandards.org. September 2006.

[4] EMV Books, 1 - Application Independent ICC to Terminal Interface Requirement and Application Selection, Book 2 Security and Key Management, Book 3 - Application Specification, Book 4 - Cardholder, Attendant, and Acquirer Interface Require, www.emvco.org

[5] T.M. Jurgensen, et al., SMART CARDS: THE DEVELOPER'S TOOLKIT, Prentice Hall, 2002.

[6] Z. Chen, JAVACARD ${ }^{\mathrm{TM}}$ TECHNOLOGY FOR SMART CARDS: ARCHITECTURE AND PROGRAMMER'S, The Java Series, Addison-Wesley, 2002
[7] M. Pasquet, "La sécurisation d'un système informatique complexe: le cas de la monétique", Habilitation à Diriger des Recherches, France, November 2008.

[8] E. Rescorla, SSL AND TLS DESIGNING AND BUILDING SECURE SYSTEMS, Addison-Wesley, 2001

[9] RFC 2246, The TLS Protocol Version 1.0, IETF, 1999

[10] RFC 2716 "PPP EAP TLS Authentication Protocol ", 1999

[11] P.Urien, G.Pujolle, "Security and Privacy for the next Wireless Generation", International Journal of Network Management, IJNM, Volume 18 Issue 2 (March/April 2008), WILEY

[12] P. Urien, "Collaboration of SSL smart cards within the WEB2 landscape", Collaborative Technologies and Systems, 2009. CTS'09. International Symposium, May 2009

[13] P.Urien, "EAP Support in Smartcard", draft-urien-eapsmartcard-20.txt, IETF DRAFT, 2011.

[14] Cakaj, S.; Shefkiu, M.; Haxha, S.; "Implementation of prepaid services in Kosovo's fixed network", ELMAR '09. International Symposium

[15] CEPSCO, Common Electronic Purse Specification, Technical Specification version 2.2, 2000 